\title{
Pharyngoplasty (Posterior Pharyngeal Flaps)
}

\section{Kai Han and Jian-min Yao}

\section{Case}

1. Medical history: A boy, 4 years old, presented with complete palate cleft.

2. Clinical manifestation: Complete palate cleft.

3. Surgical method: Lateral myocutaneous flap

4. Key points: Mucosal muscle flaps were made. Rotate the tongue-shaped flap to reduce the nasopharyngeal cavity.
5. Precautions: Make a transverse incision at the transverse ridge of the posterior pharyngeal wall and make mucosal muscle flaps on both sides with the pedicle above. Flaps were embedded in a transverse incision to form a transverse ridge and narrow the nasopharyngeal cavity.

6. Operation steps: See Fig. 12.1.
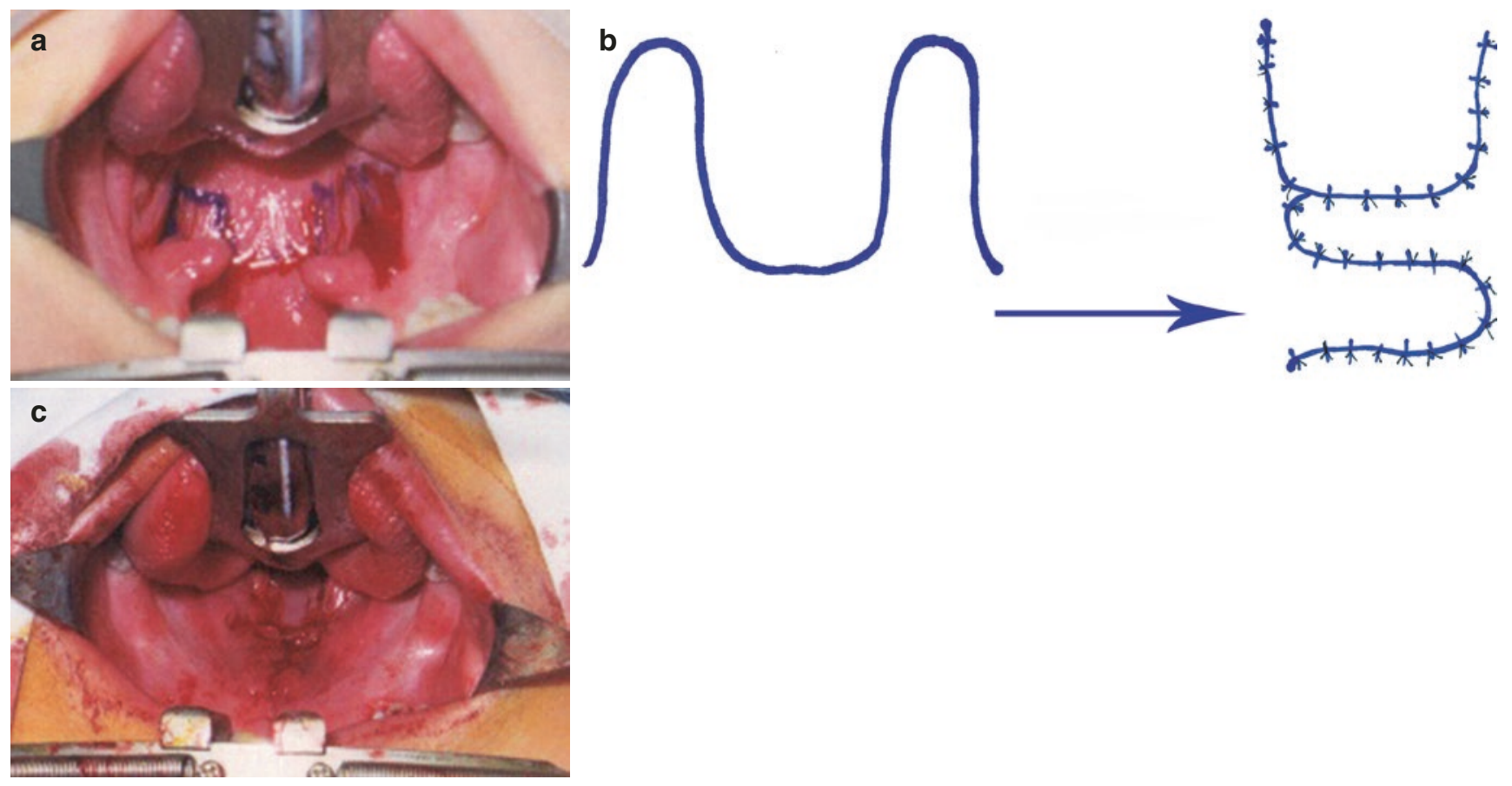

Fig. 12.1 Pharyngoplasty(postpharyngeal flap). (a) Design incision (b) Operation sketch (c) Reduction of pharyngeal cavity after operation

\section{K. Han}

Hangzhou Operation Smile Charity Hospital, Hangzhou, China

J.-m. Yao $(\bowtie)$

Hangzhou Plastic Surgery Hospital, Hangzhou, China 